\title{
Nuclear YB-1 expression as a negative prognostic marker in nonsmall cell lung cancer
}

\author{
C. Gessner*, C. Woischwill ${ }^{\#}$, A. Schumacher ${ }^{\Uparrow}$, U. Liebers ${ }^{\Uparrow}$, H. Kuhn*, P. Stiehl ${ }^{+}$, K. Jürchott ${ }^{\S}$, \\ H.D. Royer" ${ }^{\#}$ C. Witt ${ }^{\natural}$, G. Wolff ${ }^{\#}$
}

Nuclear YB-1 expression as a negative prognostic marker in nonsmall cell lung cancer. C. Gessner, C. Woischwill, A. Schumacher, U. Liebers, H. Kuhn, P. Stiehl, K. Jürchott, H.D. Royer, C. Witt, G. Wolff. (C) ERS Journals Ltd 2004.

ABSTRACT: The human Y-box binding protein, YB-1, is a multifunctional protein that regulates gene expression. Nuclear expression of YB-1 has been associated with chemoresistance and poor prognosis of tumour patients.

Representative samples from autopsied material of primary tumours from 77 patients with NSCLC were investigated by immunohistochemistry for subcellular distribution of YB-1 and p53, in order to evaluate the prognostic role of nuclear expression of YB-1.

Cytoplasmic YB-1 expression was found in all tumour samples, whereas nuclear expression was only observed in $48 \%$. There was no correlation with histological classification, clinical parameters or tumour size, stage and metastasis status. However, patients with positive nuclear YB-1 expression in tumours showed reduced survival times when compared with patients without nuclear expression. Including information about the histology and mutational status for p53 increased the prognostic value of nuclear YB-1. Patients with nuclear YB-1 expression and p53 mutations had the worst prognosis (median survival 3 months), while best outcome was found in patients with no nuclear YB-1 and wildtype p53 (median survival 15 months).

This suggests that the combined analysis of both markers allows a better identification of subgroups with varying prognosis. Nuclear expression of Y-box binding protien seems to be an independent prognostic marker.

Eur Respir J 2004; 23: 14-19.

\begin{abstract}
*Dept of Internal Medicine, Pneumology, and ${ }^{+}$Institute of Pathology, University of Leipzig, Leipzig, " Max Delbrueck Centre for Molecular Medicine, Berlin-Buch, "Dept of Pneumology, Division of Internal Medicine, Charité-Universitätsmedizin Berlin, and ${ }^{8}$ Institute of Pathology, University Hospital Charité, Humboldt University of Berlin, Berlin, Germany.
\end{abstract}

Correspondence: C. Gessner, Dept of Internal Medicine, Pulmonary unit, Johannisallee 32, 04103 Leipzig, Germany.

Fax: 493419712609

E-mail: gesc@medizin.uni-leipzig.de

Keywords: Molecular diagnosis

prognosis

survival

tumour staging

Y-box binding protein

Received: March 242003

Accepted after revision: August 212003
Lung cancer is one of the leading causes of cancer death in the Western world [1]. New chemotherapeutic drugs and multimodal treatment strategies, including first trials of gene replacement therapies, have been developed to improve prognosis [2-4]. However, the survival times of patients with nonsmall cell lung cancer (NSCLC) remain unchanged and poor. Identification of new prognostic markers to characterise lung cancer biology seems to be necessary for identification of patient subgroups to benefit from these new concepts [5].

The human Y-box binding protein, YB-1, is a multifunctional protein that regulates gene expression at both the transcriptional and translational level [6]. As a transcription factor, it binds to the $\mathrm{Y}$-box sequence in the promoter region of several genes, one example being the human multidrugresistance 1 gene encoding P-glycoprotein [7]. This P-glycoprotein, if overexpressed, seems to be associated with multidrug resistance by reducing drug accumulation in resistant cells [8]. This led to the suggestion of it being a prognostic marker for assessing the efficacy of chemotherapy [9].

The YB-1 protein is mainly located in the cytoplasm. When human cancer cells are treated with ultraviolet (UV) irradiation or anticancer agents, YB-1 translocates into the nucleus [10]. For ovarian cancer it has been shown that nuclear expression of YB-1 is associated with an acquired cisplatin resistance [11]. Transfection of human epidermoid cancer cells with a YB-1-antisense construct resulted in increased sensitivity to cisplatin, mitomycin $\mathrm{C}$ and $\mathrm{UV}$ radiation, which suggests a protective effect of YB-1 against cytotoxic effects of agents that induce cross-linking of deoxyribonucleic acid
(DNA) [12]. In addition, YB-1 interacts with the tumour suppressor p53, which is also activated by genotoxic stress and DNA damage, supporting a functional role in the DNA damage response as well as in DNA repair. Furthermore, this interaction has been demonstrated to mutually modulate the DNA-binding function of both proteins, thereby altering the expression of genes containing YB-1 or p53-binding sites as exemplified by the multidrug-resistance 1 gene [13].

An association between nuclear expression of YB-1 and high P-glycoprotein levels has been demonstrated in further clinical investigations for breast cancer [14], human osteosarcoma and synovial sarcoma [15, 16]. Moreover, KAMURA et al. [17] reported YB-1 as a useful prognostic marker in ovarian adenocarcinoma. For NSCLC, SHIBAHARA et al. [7] reported that nuclear expression of YB-1 may be an important factor for disease progression. They found a poorer prognosis for those patients with nuclear compared with patients with cytoplasmatic YB-1 expression, especially in squamous cell carcinoma.

In this study, therefore, the prognostic relevance of nuclear YB-1 expression was investigated in NSCLC with respect to p53 [13].

\section{Patients and methods}

\section{Patients}

Representative tumour samples $(n=4)$ were obtained at autopsy from primary tumours from 77 consecutive patients 
with NSCLC (59 male, 18 female, median age 65 ys, range 46-83 yrs). All of the patients studied died from the tumour and analysis was performed retrospectively. All patients were treated between 1991-1996 at the University of Leipzig Medical Centre and associated academic hospitals. The histological tumour classification was performed by two pathologists according to World Health Organization guidelines [18]. The tumour stage was determined at the time of diagnosis according to the revised tumour, node, metastasis (TNM) staging system of lung cancer [19]. Survival times and clinical data were retrospectively assessed (table 1).

\section{Western blot analysis}

For Western blot analysis, whole cell lysates were prepared from Hela cells (No. CCL-2; American Type Culture Collection, Manassas, VA, USA) by trichloracetic acid precipitation and immunoblotting was performed according to standard procedures using a 1:2,500 dilution of a YB-1 antiserum. This polyclonal peptide antiserum does not cross-react with other Y-box proteins and was affinity purified using the same peptide [6]. The blocking peptide was used in 10-fold molar excess.

\section{Immunohistochemical analysis of p53 and Y-box binding protein}

The protein expression of p53 and YB-1 was analysed by immunohistochemistry using $2-\mu \mathrm{m}$ formalin-fixed, paraffinembedded tissue slices. For antigen demasking, slices were boiled after deparaffination for $10 \mathrm{~min}$ in citrate buffer (0.01 M, pH 6.0). After blocking with fish serum (SEA BLOCK $^{\mathrm{TM}}$; Pierce, Rockford, IL, USA), slices were incubated for $60 \mathrm{~min}$ with either the primary monoclonal anti-p53 antibody (DO-1, dilution 1:50; BDBiosciences, Heidelberg, Germany) or with the polyclonal YB-1 antiserum (dilution 1:200). The bound primary antibodies were visualised by

Table 1.-Tumour parameters

$\begin{array}{lc}\text { T-stage of primary tumour } & \\ \text { T1 } & 11(14) \\ \text { T2 } & 27(35) \\ \text { T3 } & 19(25) \\ \text { T4 } & 20(26) \\ \text { Lymph node metastases } & \\ \text { N0 } & 20(26) \\ \text { N1 } & 15(20) \\ \text { N2 } & 21(27) \\ \text { N3 } & 21(27) \\ \text { Distant metastasis } & \\ \text { M0 } & 38(49) \\ \text { M1 } & 39(51) \\ \text { Tumour stage groups } & \\ \text { IA } & 5(7) \\ \text { IB } & 8(10) \\ \text { IIA } & 3(4) \\ \text { IIB } & 7(9) \\ \text { IIIA } & 8(10) \\ \text { IIIB } & 7(9) \\ \text { IV } & 39(51) \\ \text { Histological classification } & \\ \text { Adenocarcinoma } & 26(34) \\ \text { Bronchioloalveolar carcinoma } & 4(5) \\ \text { Squamous cell carcinoma } & 40(52) \\ \text { Large cell carcinoma } & 7(9) \\ \end{array}$

Data are presented as n (\%) unless otherwise stated. biotin-conjugated secondary antibodies and peroxidase-conjugated streptavidin (Jackson Immuno Research Laboratories, West Grove, ME, USA), using metal-enhanced 3,3'-diaminobenzidine as a substrate (Pierce). Slices were counterstained with haematoxylin.

Slices were analysed in a blinded fashion by two observers who were unaware of clinical and histological data. Four high power fields $(400 \times)$ were evaluated for localisation and percentage of positive cells $(0-100 \%$ in $5 \%$ steps $)$. Samples were considered as positive if $>10 \%$ of tumour cells showed moderate or intense nuclear staining.

\section{Mutation analysis of p53}

DNA was extracted from $30 \mu \mathrm{m}$ slices of paraffin-embedded tissue. Genomic DNA was isolated after deparaffination and rehydration using the InViSorb ${ }^{\mathrm{TM}}$ Genomic DNA Kit II (InViTek, Berlin, Germany).

For detection of mutations in the DNA-binding region of p53 single-strand conformation polymorphism (SSCP), polymerase chain reaction (PCR) analysis was performed [20]. It was used as an established standard method, although SSCP may miss some of the p53 mutations and therefore patients classified as wildtype for p53 may possibly carry an undetected mutation. Template DNA was subjected to PCR using oligonucleotide primer pairs for amplification of exons 5-8. The amplified fragments were analysed on a $10 \%$ nondenaturing polyacrylamide gel with silver staining using a DNA silverstaining kit (Amersham Pharmacia Biotech, Freiburg, Germany) according to the manufacturer.

\section{Statistical analysis}

Overall survival was estimated by the Kaplan-Meier productlimit method. Statistical comparison was performed by means of the Log rank Mantel-Cox test. Multivariate survival analysis was performed by Cox regression. Most biological and pathological variables were used as dichotomised (categorical) variables. Patients were stratified in positive (p53pos, YB-1pos) versus negative (p53neg, YB-1neg). p53 genetic status was subdivided in p53 wildtype (p53wt) versus p53 mutated (p53mut). The cut-off value for immunohistochemical data of p53 and YB-1 was set as $10 \%$ positively stained cells, i.e. $\geqslant 10 \%$ stained cells in a tumour was considered to be a positive result, $<10 \%$ stained cells was considered negative [21].

For correlation of p53 expression and YB-1 nuclear localisation with tumour classification, as well as with clinical parameters, Pearson's Chi-squared test was applied. Fisher's exact test was applied in the survival analysis to test for the distribution of patients with different therapies in the positive and negative subgroups for $\mathrm{p} 53$ and YB-1.

\section{Results}

\section{Follow-up}

At the time of diagnosis $51 \%$ of patients (39 of 77) were in tumour stage IV, while only $49 \%$ were stages I-III (38 of 77). Twelve patients received best supportive care only due to their advanced stage, 12 were treated by surgical resection, 18 by chemotherapy and 13 by radiation therapy. Nine patients underwent surgery and adjuvant chemotherapy, four neoadjuvant surgery and radiotherapy, and six received chemotherapy and radiotherapy. Three patients were treated with 


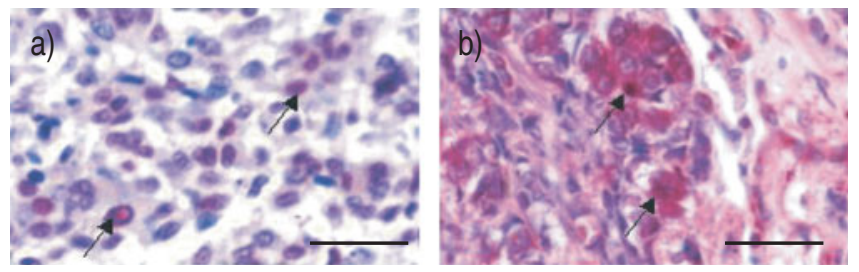

Fig. 1.-Immunohistochemistry for a) p53 and b) YB-1 in a representative sample of nonsmall cell lung cancer patients. Arrows indicate cells a) overexpressing p53 or b) with nuclear localisation of YB-1. Scale bars $=250 \mu \mathrm{m}$

surgery, adjuvant chemotherapy, and radiotherapy. These patients were equally distributed in the positive and negative subgroups for YB-1 ( $>>0.21)$, and p53 ( $\mathrm{p}>0.67)$, as well as in the $\mathrm{p} 53$ mutation status subgroups $(\mathrm{p}>0.99)$.

Median overall survival was 8 months. The overall 1- and 2-yr survival rates (including the 30-day perioperative mortality of patients treated by surgery) were $34 \%$ and $12 \%$, respectively.

\section{Immunohistochemical analysis of p53 expression}

Overexpression of p53 protein (fig. 1a) was found in 41 of 77 cases with a mean number of (mean \pm SEM) $62 \pm 3 \%$ of p53expressing cells. Twenty-nine of 77 tumours showed $0 \%$, one of 77 showed $5 \%$, and six of 77 samples showed $10 \%$ of cells positively stained for $\mathrm{p} 53$.

To analyse the clinical relevance of $\mathrm{p} 53$, the protein expression status was correlated with tumour and clinical parameters. Neither tumour parameters (size, lymph node metastasis status, distant metastasis status, tumour stage, grading and histological classification) nor age, sex or therapy status showed a significant correlation with p53 expression.

\section{Immunohistochemical analysis of Y-box binding protein expression}

The specificity of the YB-1 antiserum used for immunohistochemistry was shown by Western blot analysis of Hela cells (fig. 2). Using the antiserum the $50 \mathrm{kD} \mathrm{YB-1}$ protein was detected in the cells (lane 1), whereas addition of the corresponding blocking peptide abolished the signal (lane 2).

YB-1 expression in the cytoplasm was found in all of the 77 NSCLC tumour samples. Nuclear expression of YB-1 (fig. 1b) was observed in 37 of 77 patients while in 40 of 77 patients no cells with nuclear YB-1 expression were found. The fraction of cells with positive nuclear YB-1 expression was $50 \pm 3 \%$ in these 37 NSCLC patients.

Neither histological classification nor clinical parameters showed a significant correlation with nuclear YB-1 status. Nor was there a correlation between tumour size, stage, or lymph node metastasis status and nuclear YB-1 expression.

However, a significant correlation of distant metastasis status and of grading type was found with nuclear YB-1 expression. Tumours with distant metastasis were positive for nuclear expression of YB-1 in $61 \%$ and negative in $39 \%$ of the cases, while tumours without distant metastasis were positive for nuclear YB-1 expression in $36 \%$ and negative in $64 \%$ $(\mathrm{p}<0.05)$.

With respect to tumour grading, nuclear staining for YB-1 was observed in $36 \%$ of samples of grading type $\mathrm{G} 2$ and $61 \%$ of samples in G3, while nuclear staining was absent in $64 \%$ of grading type $\mathrm{G} 2$ and in $39 \%$ of samples in $\mathrm{G} 3(\mathrm{p}<0.03)$. These data indicate that nuclear expression of YB-1 may be associated with tumour tissue of lower histological differentiation.

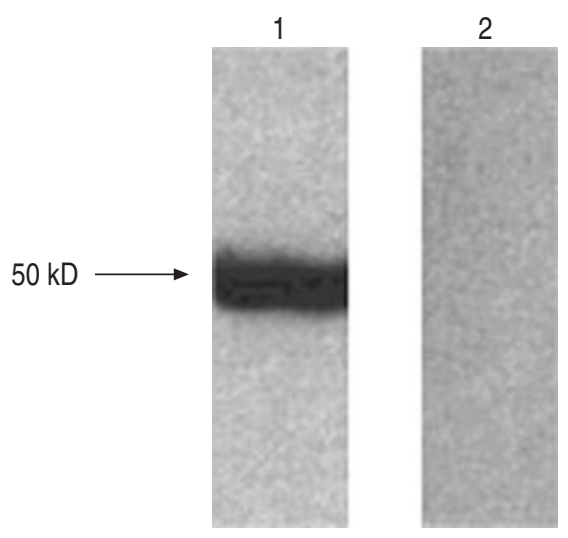

Fig. 2.-Western blot analysis showing the specificity of the YB-1 antiserum. Lane 1 indicates YB-1 antiserum and lane 2 indicates YB1 antiserum and peptide.

Grading type G1 and G4 were not represented in the tumour samples.

\section{Analysis of p53 mutations}

In 40 of 77 primary tumours $(53 \%)$, mutations in the p53 gene were found. Altogether 44 mutations were detected: 10 in exon 5, seven in exon 6,18 in exon 7 , and nine in exon 8 . Four primary tumours showed mutations in two exons. Tumours with mutated p53 had no significantly increased protein expression. Of the cases, 58\% with p53 mutations versus $49 \%$ of the cases without $\mathrm{p} 53$ mutation stained positive for $\mathrm{p} 53$.

\section{Correlation of histology, p53 status and nuclear localisation of Y-box binding protein with survival}

To determine the prognostic value of p53 protein expression and mutation status (exons 5-8), as well as nuclear expression of YB-1, a univariate survival analysis was carried out.

There was no prognostic impact of p53 protein expression (p53pos median survival 6 months, p53neg median survival 9 months; $\mathrm{p}=0.56$ ) as well as $\mathrm{p} 53$ mutational status ( $\mathrm{p} 53 \mathrm{wt}$ median survival 10 months, p53mut median survival 5 months; $\mathrm{p}=0.16)$.

Patients with positive nuclear YB-1 expression in tumours showed reduced survival times (median survival 5 months) when compared with patients without nuclear expression (median survival 13 months, $\mathrm{p}<0.0001$; fig. 3). Furthermore, an artefactual effect due to a heterogeneous groups of patients was excluded by comparing survival times for subgroups, such as stages I-III, stage IV, and therapeutic intervention (table 2).

Based on the data of Окамото et al. [13], who described a direct interaction of $\mathrm{p} 53$ with YB-1, a further survival analysis for the $\mathrm{p} 53 \mathrm{wt} / \mathrm{p} 53$ mut and $\mathrm{p} 53$ pos/p53neg subgroups, and YB-1 nuclear expression was carried out (table 2). The best outcomes were seen in the $\mathrm{p} 53 \mathrm{wt} / \mathrm{YB}-1$ neg group (median survival 15 months; fig. 4), and in the p53neg/YB-1neg group (median survival 12 months; fig. 5).

Since Shibahara et al. [7] found differences in prognosis with respect to histology, survival times for adenocarcinoma and squamous cell carcinoma were also compared. In both histological subgroups of tumours, patients with no nuclear YB-1 expression survived longer than those with nuclear 


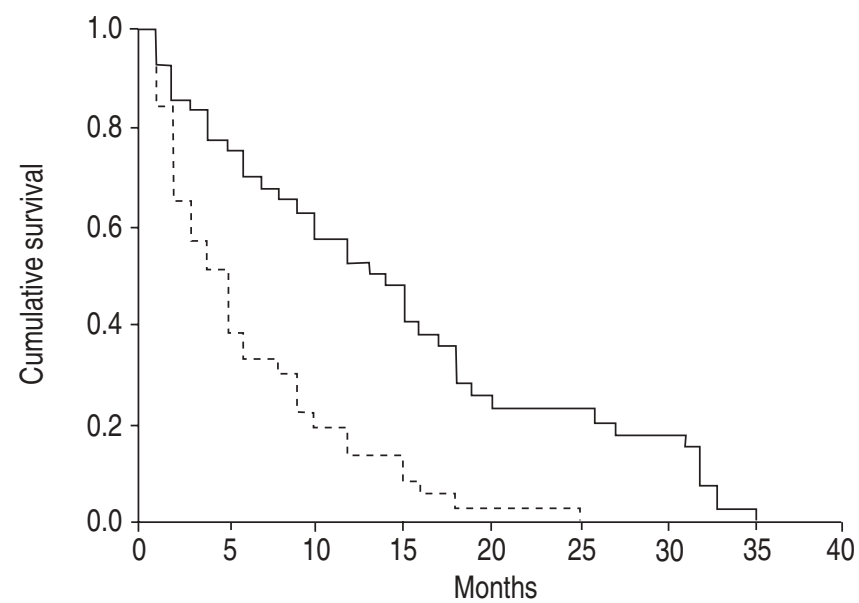

Fig. 3.-Survival analysis for nuclear YB-1 expression. Kaplan-Meier analysis of overall survival in the group with nuclear YB-1 expression $(--; n=37)$ and in the group without nuclear YB-1 expression (-; $\mathrm{n}=40, \log$ rank Mantel-Cox $\mathrm{p}<0.0001)$.

YB-1 expression (adenocarcinoma YB-1pos median survival 5 months, YB-1neg median survival 14 months, $\mathrm{p}<0.009$; squamous cell carcinoma YB-1pos median survival 3 months, YB-1 neg median survival 15 months; $\mathrm{p}<0.0005$ ). Owing to the small number of patients with large cell carcinoma $(n=7)$ and bronchioloalveolar carcinoma $(n=4)$, survival times were not compared for these groups.

To further corroborate the prognostic importance of nuclear YB-1 expression as an independent factor, a multivariate analysis was performed. This multivariate survival analysis showed no significant influence of potential prognostic factors (TNM, grading, p53, histopathology, treatment) other than nuclear YB-1 expression, suggesting this as an independent prognostic factor (table 3 ).

\section{Discussion}

The data of this analysis showed a prognostic value for nuclear YB-1 expression in patients with NSCLC. Note that the specimens for this study were obtained from autopsied material from patients that died of their tumour. Therefore, it cannot be ruled out that survival times may be biased towards shorter survival. This will, however, not influence/affect the principal result of this investigation. The observation, a prognostic impact of nuclear YB-1 expression, also held true

Table 2.-Survival analysis for different subgroups

\begin{tabular}{llcc}
\hline Groups & \multicolumn{3}{c}{ Median survival months } \\
\cline { 2 - 4 } & $\begin{array}{c}\text { Nuclear } \\
\text { expression }\end{array}$ & $\begin{array}{c}\text { No nuclear } \\
\text { expression }\end{array}$ & $\mathrm{p}$-value \\
\hline YB-1 & $5(37)$ & $13(40)$ & $\mathrm{p}<0.0001$ \\
Stages I-III group & $8(14)$ & $17(24)$ & $\mathrm{p}<0.003$ \\
Stage IV group & $2(23)$ & $6(16)$ & $\mathrm{p}<0.013$ \\
Therapy group & $5(29)$ & $15(36)$ & $\mathrm{p}<0.005$ \\
Best supportive care & $2(8)$ & $3(4)$ & $\mathrm{NS}$ \\
p53wt and & $5(14)$ & $15(23)$ & \\
p53mut & $3(23)$ & $13(17)$ & $\mathrm{p}<0.0001$ \\
p53pos and & $3(17)$ & $4(24)$ & \\
p53neg & $8(20)$ & $12(16)$ & $\mathrm{p}<0.0001$ \\
\hline
\end{tabular}

Data are presented as months survival (total $\mathrm{n}$ ). $\mathrm{p}$-value derived from Log rank-Mantel-Cox-Test. NS: nonsignificant.

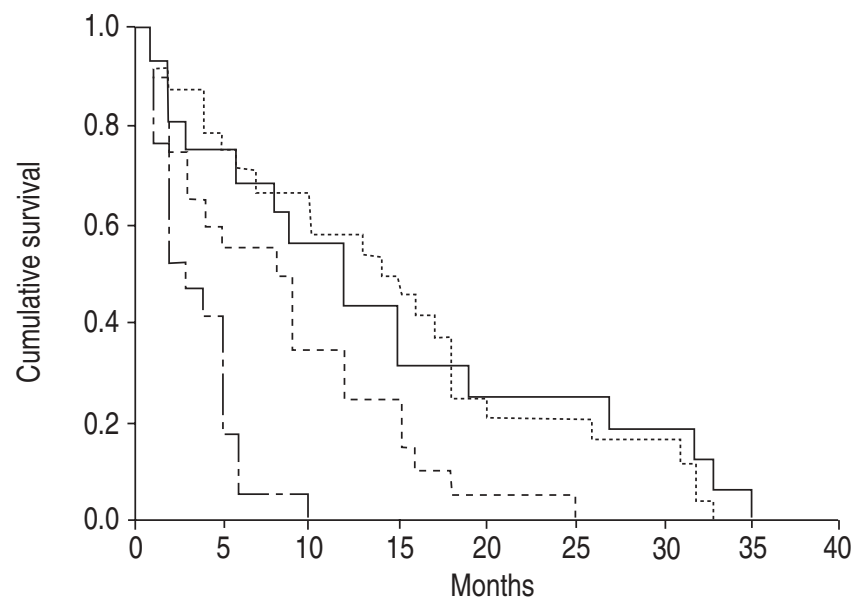

Fig. 4.-Combination of survival analysis of nuclear YB-1 expression with p53 mutational status. —_ no nuclear YB-1 or p53 expression $(n=16) ; \cdots \cdots \cdots \cdot$. no nuclear YB-1 expression but p53 expression $(n=24)$; - - -: nuclear YB-1 expression but no p53 expression $(n=20) ;-\cdot-\cdot$ nuclear YB-1 and p53 expression $(n=17)$.

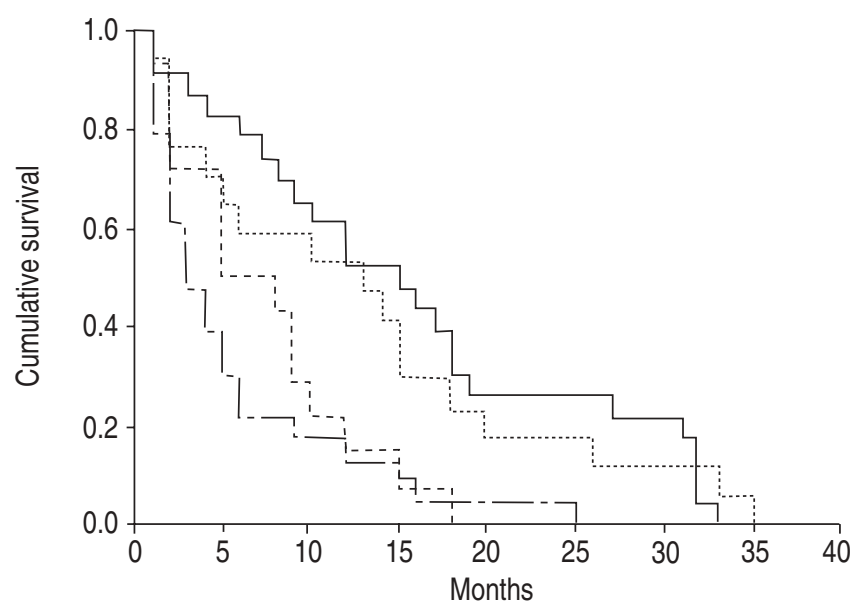

Fig. 5.-Combination of survival analysis of nuclear YB-1 expression with p53 expression status. — : no nuclear YB-1 expression and wildtype p53 $(n=23) ; \cdots \cdots \cdots \cdots$ : no nuclear YB-1 expression and mutant p53 $(n=17)$; - - -: nuclear YB-1 expression and wildtype p53 $(n=14)$; $--\because$ nuclear YB-1 expression and mutant p53 $(n=23)$.

Table 3.-Multivariate survival analysis performed by Cox regression

\begin{tabular}{lccc}
\hline Parameter & Relative Risk & $95 \%$ CI & p-value \\
\hline Nuclear YB-1 expression $^{\#}$ & 2.34 & $1.29-4.25$ & 0.005 \\
T-staging\# $^{\text {N-staging }}$ & 1.01 & $0.52-1.94$ & 0.982 \\
M-staging & 1.97 & $1.00-3.90$ & 0.051 \\
Treatment & 1.85 & $0.98-3.50$ & 0.057 \\
Histopathology & 0.60 & $0.27-1.30$ & 0.192 \\
$\quad$ Adenocarcinoma & 1.00 & & 0.384 \\
$\quad$ Squamous cell carcinoma & 1.11 & $0.64-1.93$ & 0.711 \\
$\quad$ Others & 1.84 & $0.76-4.47$ & 0.180 \\
p53 expression & 1.58 & $0.94-2.63$ & 0.081 \\
p53 mutational status & 1.12 & $0.68-1.81$ & 0.682 \\
\hline
\end{tabular}

CI: confidence interval. ${ }^{\#}$ : T-stage 1 and 2 versus T-stage 3 and $4 ;{ }^{\circ}: \mathrm{N}$ stage 0 and 1 versus $\mathrm{N}$-stage 2 and $3 ;^{+}$: best supportive care versus treatment. $\$$ : bronchioloalveolar carcinoma and large cell carcinoma. 
when YB-1 subgroups were analysed to exclude a delusional effect caused by the heterogeneity of the analysed group (table 2), suggesting YB-1 as an independent prognostic marker. p53 overexpression as well as p53 mutational status alone had no significant influence on survival. This result, a weak prognostic significance of p53 alterations in lung cancer, is in concordance with findings of other groups [22, 23]. When adding the information about the expression status of p53 to the nuclear expression status of YB-1, however, the dichotomy of the negative prognostic relevance of nuclear YB-1 expression becomes even more striking. Patients with nuclear YB-1 expression and p53 mutations had the worst prognosis (median survival 3 months) while the best outcome was found for patients with no nuclear YB-1 expression and wildtype p53 (median survival 15 months). Since Окамото et al. [13] had previously demonstrated that YB-1 directly interacts with p53 and that wildtype p53 is required to modulate the transcriptional activity of YB-1, this result is not unexpected. Taken together these data suggest that combined analysis of both markers may provide improved accuracy for identification of subgroups with varying prognosis.

A prognostic impact of nuclear YB-1 expression was also described for other cancers such as human breast carcinoma [6], ovarian serous adenocarcinoma [17] and synovial sarcoma [16]. Nuclear localisation of YB-1 was suggested as a prognostic marker for multidrug resistance in osteosarcoma [15]. In NSCLC, SHIBAHARA et al. [7] observed a poorer prognosis for patients with nuclear YB-1 expression compared with cytoplasmatic YB-1 expression $(\mathrm{p}=0.049)$. However, in their analysis of histological subgroups this prognostic significance was only true for squamous cell carcinoma and not for adenocarcinomas [7]. Analysis of the different histological subgroups did not point out an influence of histology on the prognostic outcome. In adenocarcinoma, as well as in squamous cell carcinoma, the present authors found that nuclear YB-1 expression was a negative prognostic marker. A possible explanation for these divergent observations could be differences in the patients cohorts. While in the present study 39 patients presented with tumour stage IV, only seven patients were in stage IV in the analysis of SHIBAHARA et al. [7].

Correlation of clinicopathological markers and nuclear YB-1 expression revealed a correlation of nuclear YB-1 expression with distant metastasis status as well as with tumour grading. For both parameters, distant metastasis and lower histological grading, an association with nuclear YB-1 expression was found more often. The same result was found by SHIBAHARA et al. [7] for grading. However, these authors also describe a correlation of nuclear YB-1 expression with tumour stage, $\mathrm{T}$ factor, and $\mathrm{N}$ factor, which, in addition, varied with the different histological forms of NSCLC. This discordance in results could be caused by the smaller number of patients investigated in the present study and with differences in the patient groups concerning tumour stage.

Nuclear expression of YB-1 was observed in human cancer cells in response to genotoxic stress such as UV radiation or chemotherapy [12]. Further investigations of ISE et al. [24] suggested that YB-1 can function as a recognition protein for cisplatin-damaged DNA. YB-1 regulates the expression of the multidrug-resistance gene MDR1 which encodes P-glycoprotein. This protein plays an important role in the development of a multidrug-resistant tumour phenotype [25]. In the investigation of JANZ et al. [25], the prognostic impact of nuclear YB-1 expression was evaluated in dependence of chemotherapy in two patient groups, a low-risk and a high-risk breast cancer group. In both groups, high nuclear YB-1 expression in breast cancer tissue was associated with an unfavourable clinical course. The authors concluded that YB-1 expression is associated with clinical drug resistance as well as tumour aggressiveness [25]. In concordance with the results of this study, nuclear YB-1 expression is an independent prognostic marker for NSCLC. Prognostic markers such as YB-1, p16 or Bcl2associated $\mathrm{X}$ protein [26] are prerequisites for an adequate risk group assessment in order to individualise therapy concepts in NSCLC.

Since prognosis in nonsmall cell lung cancer in general is rather poor, molecular diagnosis should be added to evaluate useful predictive markers, in particular for chemosensitivity. Based on the data of SHIBAHARA et al. [7] and of the present study concerning the prognostic influence of nuclear Y-box binding protein expression in nonsmall cell lung cancer, a controlled prospective study is warranted to clarify differences between both investigations and to validate a prognostic value of nuclear Y-box binding protein expression in relation to multimodal therapy concepts including chemotherapy, radiation, surgery and combined concepts.

\section{References}

1. Parker SL, Tong T, Bolden S, Wingo PA. Cancer statistics 1997. CA Cancer J Clin 1997; 47: 5-27.

2. Esnaola NF, Lazarides SN, Mentzer SJ, Kuntz KM. Outcomes and cost-effectiveness of alternative staging strategies for non-small-cell lung cancer. J Clin Oncol 2002; 20: 263-273.

3. Lacquet LK. The present status of surgery for lung cancer. Acta Chir Belg 1996; 96: 245-251.

4. Muller H, Hilger R. Curative and palliative aspects of regional chemotherapy in combination with surgery. Support Care Cancer 2003; 11: 1-10.

5. Brundage MD, Davies D, Mackillop WJ. Prognostic factors in non-small cell lung cancer: a decade of progress. Chest 2002; 122: 1037-1057.

6. Bargou RC, Jurchott $\mathrm{K}$, Wagener $\mathrm{C}$, et al. Nuclear localization and increased levels of transcription factor YB-1 in primary human breast cancers are associated with intrinsic MDR1 gene expression. Nat Med 1997; 3: 447-450.

7. Shibahara K, Sugio K, Osaki T, et al. Nuclear expression of the Y-box binding protein, YB-1, as a novel marker of disease progression in non-small cell lung cancer. Clin Cancer Res 2001; 7: 3151-3155.

8. Gottesman MM, Pastan I. Biochemistry of multidrug resistance mediated by the multidrug transporter. Annu Rev Biochem 1993; 62: 385-427.

9. Beck WT, Grogan TM, Willman CL, et al. Methods to detect P-glycoprotein-associated multidrug resistance in patients' tumors: consensus recommendations. Cancer Res 1996; 56: 3010-3020.

10. Koike K, Uchiumi T, Ohga T, et al. Nuclear translocation of the Y-box binding protein by ultraviolet irradiation. FEBS Lett 1997; 417: 390-394.

11. Yahata $\mathrm{H}$, Kobayashi $\mathrm{H}$, Kamura $\mathrm{T}$, et al. Increased nuclear localization of transcription factor YB-1 in acquired cisplatin-resistant ovarian cancer. J Cancer Res Clin Oncol 2002; 128: 621-626.

12. Ohga T, Koike $\mathrm{K}$, Ono $\mathrm{M}$, et al. Role of the human $\mathrm{Y}$ box-binding protein YB-1 in cellular sensitivity to the DNAdamaging agents cisplatin, mitomycin $\mathrm{C}$, and ultraviolet light. Cancer Res 1996; 56: 4224-4228.

13. Okamoto T, Izumi H, Imamura $\mathrm{T}$, et al. Direct interaction of p53 with the Y-box binding protein, YB-1: a mechanism for regulation of human gene expression. Oncogene 2000; 19: 6194-6202.

14. Bargou RC, Daniel PT, Mapara MY, et al. Expression of the bcl-2 gene family in normal and malignant breast tissue: low bax-alpha expression in tumor cells correlates with resistance towards apoptosis. Int J Cancer 1995; 60: 854-859.

15. Oda Y, Sakamoto A, Shinohara N, et al. Nuclear expression of YB-1 protein correlates with P-glycoprotein expression in human osteosarcoma. Clin Cancer Res 1998; 4: 2273-2277. 
16. Oda Y, Ohishi Y, Saito T, et al. Nuclear expression of Y-box-binding protein-1 correlates with P-glycoprotein and topoisomerase II alpha expression, and with poor prognosis in synovial sarcoma. J Pathol 2003; 199: 251-258.

17. Kamura T, Yahata H, Amada S, et al. Is nuclear expression of Y box-binding protein-1 a new prognostic factor in ovarian serous adenocarcinoma? Cancer 1999; 85: 2450-2454.

18. Brambilla E, Travis WD, Colby TV, Corrin B, Shimosato Y. The new World Health Organization classification of lung tumours. Eur Respir J 2001; 18: 1059-1068.

19. Mountain CF. Revisions in the International System for Staging Lung Cancer. Chest 1997; 111: 1710-1717.

20. Orita M, Iwahana H, Kanazawa H, Hayashi K, Sekiya T. Detection of polymorphisms of human DNA by gel electrophoresis as single-strand conformation polymorphisms. Proc Natl Acad Sci USA 1989; 86: 2766-2770.

21. Sturm I, Kohne CH, Wolff G, et al. Analysis of the p53/BAX pathway in colorectal cancer: low BAX is a negative prognostic factor in patients with resected liver metastases. J Clin Oncol 1999; 17: 1364-1374.
22. Steels E, Paesmans M, Berghmans T, et al. Role of p53 as a prognostic factor for survival in lung cancer: a systematic review of the literature with a meta-analysis. Eur Respir $J$ 2001; 18: 705-719.

23. Dworakowska D, Gozdz S, Jassem E, et al. Prognostic relevance of proliferating cell nuclear antigen and p53 expression in non-small cell lung cancer. Lung Cancer 2002; 35: $35-41$.

24. Ise $\mathrm{T}$, Nagatani $\mathrm{G}$, Imamura $\mathrm{T}$, et al. Transcription factor Y-box binding protein 1 binds preferentially to cisplatinmodified DNA and interacts with proliferating cell nuclear antigen. Cancer Res 1999; 59: 342-346.

25. Janz M, Harbeck N, Dettmar P, et al. Y-box factor YB-1 predicts drug resistance and patient outcome in breast cancer independent of clinically relevant tumor biologic factors HER2, uPA and PAI-1. Int J Cancer 2002; 97: 278-282.

26. Gessner C, Liebers U, Kuhn H, et al. BAX and p16INK4A are independent positive prognostic markers for advanced tumour stage of nonsmall cell lung cancer. Eur Respir J 2002; 19: $134-140$. 